\title{
DUAL WAVELETS ASSOCIATED WITH NONUNIFORM MRA
}

\author{
MOHAMMAD YOUNUS BHAT
}

\begin{abstract}
A generalization of Mallat's classical multiresolution analysis, based on the theory of spectral pairs, was considered in two articles by Gabardo and Nashed. In this setting, the associated translation set is no longer a discrete subgroup of $\mathbb{R}$ but a spectrum associated with a certain one-dimensional spectral pair and the associated dilation is an even positive integer related to the given spectral pair. In this paper, we construct dual wavelets which are associated with Nonuniform Multiresolution Analysis. We show that if the translates of the scaling functions of two multiresolution analyses are biorthogonal, then the associated wavelet families are also biorthogonal. Under mild assumptions on the scaling functions and the wavelets, we also show that the wavelets generate Riesz bases.
\end{abstract}

\section{Introduction}

Multiresolution analysis (MRA) is an important mathematical tool since it provides a natural framework for understanding and constructing discrete wavelet systems. A multiresolution analysis is an increasing family of closed subspaces $\left\{V_{j}: j \in \mathbb{Z}\right\}$ of $L^{2}(\mathbb{R})$ such that $\bigcap_{j \in \mathbb{Z}} V_{j}=\{0\}, \bigcup_{j \in \mathbb{Z}} V_{j}$ is dense in $L^{2}(\mathbb{R})$ and which satisfies $f \in V_{j}$ if and only if $f(2 \cdot) \in V_{j+1}$. Furthermore, there exists an element $\varphi \in V_{0}$ such that the collection of integer translates of function $\varphi,\{\varphi(\cdot-k): k \in \mathbb{Z}\}$ represents a complete orthonormal system for $V_{0}$. The function $\varphi$ is called the scaling function or the father wavelet. The concept of multiresolution analysis has been extended in various ways in recent years. These concepts are generalized to $L^{2}\left(\mathbb{R}^{d}\right)$, to lattices different from $\mathbb{Z}^{d}$, allowing the subspaces of multiresolution analysis to be generated by Riesz basis instead of orthonormal basis, admitting a finite number of scaling functions, replacing the dilation factor 2 by an integer $M \geq 2$ or by an expansive matrix $A \in G L_{d}(\mathbb{R})$ as long as $A \subset A \mathbb{Z}^{d}$. But in all these cases, the translation set is always a group. Recently, Gabardo and Nashed in [9] defined a multiresolution analysis associated with a translation set $\{0, r / N\}+2 \mathbb{Z}$, where $N \geq 1$ is an integer, $1 \leq r \leq 2 N-1, r$ is an odd integer and $r, N$ are relatively prime, a

Received November 09, 2017, accepted August 20, 2018.

2010 Mathematics Subject Classification. 42C40, 42C15, 43A70, 11 S85.

Key words and phrases. Nonuniform, biorthogonal, scaling function, Fourier transform. 
discrete set which is not necessarily a group. They call this an NUMRA. As, the case $N=1 \mathrm{re-}$ duces to the standard definition of MRA with dyadic dilation. NUMRA with multiplicity $D$, is called NUMRA-D that generalizes a particular case of a result of Calogero and Garrigos [3] on biorthogonal MRA's of multiplicity $D$ in nonstandard setup. A study with respect to NUMRA has been done by many authors in the references $[8,9,10,11,12,15,16,17]$.

The concept of biorthogonal wavelets plays an important role in applications. We refer to $[1,2,4,5,13]$ for various aspects of this theory on $\mathbb{R}$. For the higher dimensional situation on $\mathbb{R}^{n}$, we refer to the articles $[4,5,6,7,14]$.

In this article we construct dual wavelets which are associated with Nonuniform Multiresolution Analysis. We show that if $\varphi$ and ËIJ $\tilde{\varphi}$ are the scaling functions of two multiresolution analyses (MRAs) such that their translates are biorthogonal, then the associated families of wavelets are also biorthogonal. Under mild decay conditions on the scaling functions and the wavelets, we also show that the wavelets generate Riesz bases for $L^{2}(\mathbb{R})$.

The article is organized as follows. In Section 2, we give a brief introduction about nonuniform wavelets on $\mathbb{R}$. In Section 3, we find necessary and sufficient conditions for the translates of a function to form a Riesz basis for its closed linear span. In the last section, we prove that the wavelets associated with dual MRAs are biorthogonal and generate Riesz bases for $L^{2}(\mathbb{R})$.

\section{Preliminaries}

Definition 2.1. A multiresolution analysis (MRA) of $L^{2}(\mathbb{R})$ is a sequence of closed subspaces $\left\{V_{j}: j \in \mathbb{Z}\right\}$ of $L^{2}(\mathbb{R})$ satisfying the following properties:

(a) $V_{j} \subset V_{j+1}$ for all $j \in \mathbb{Z}$;

(b) $\cup_{j \in \mathbb{Z}} V_{j}$ is dense in $L^{2}(\mathbb{R})$;

(c) $\bigcap_{j \in \mathbb{Z}} V_{j}=\{0\}$;

(d) $f(x) \in V_{j}$ if and only if $f(2 x) \in V_{j+1}$ for all $j \in \mathbb{Z}$;

(e) There is a function $\varphi \in V_{0}$, called the scaling function, such that $\{\varphi(x-k): k \in \mathbb{Z}\}$ forms an orthonormal basis for $V_{0}$.

According to the standard scheme for construction of MRA-based wavelets, for each $j$, we define a space $W_{j}$ (wavelet space) as the orthogonal complement of $V_{j}$ in $V_{j+1}$, i.e., $V_{j+1}=$ $V_{j} \oplus W_{j}, j \in \mathbb{Z}$, where $W_{j} \perp V_{j}, j \in \mathbb{Z}$. It is not difficult to see that

$$
f(x) \in W_{j} \quad \text { if and only if } f(2 x) \in W_{j+1}, \quad j \in \mathbb{Z} .
$$

Moreover, they are mutually orthogonal, and we have the following orthogonal decompositions:

$$
L^{2}(\mathbb{R})=\bigoplus_{j \in \mathbb{Z}} W_{j}=V_{0} \oplus\left(\bigoplus_{j \geq 0} W_{j}\right) .
$$


For an integer $N \geq 1$ and an odd integer $r$ with $1 \leq r \leq 2 N-1$ such that $r$ and $N$ are relatively prime, we define

$$
\Lambda=\left\{0, \frac{r}{N}\right\}+2 \mathbb{Z}=\left\{\frac{r k}{N}+2 n: n \in \mathbb{Z}, k=0,1\right\} .
$$

It is easy to verify that $\Lambda$ is not necessarily a group nor a uniform discrete set, but is the union of $\mathbb{Z}$ and a translate of $\mathbb{Z}$. Moreover, the set $\Lambda$ is the spectrum for the spectral set $\Gamma=\left[0, \frac{1}{2}\right) \cup$ $\left[\frac{N}{2}, \frac{N+1}{2}\right)$ and the pair $(\Lambda, \Gamma)$ is called a spectral pair $[9,10]$.

Definition 2.2. For an integer $N \geq 1$ and an odd integer $r$ with $1 \leq r \leq 2 N-1$ such that $r$ and $N$ are relatively prime, an associated nonuniform multiresolution analysis is a sequence of closed subspaces $\left\{V_{j}: j \in \mathbb{Z}\right\}$ of $L^{2}(\mathbb{R})$ such that the following properties hold:

(a) $V_{j} \subset V_{j+1}$ for all $j \in \mathbb{Z}$;

(b) $\cup_{j \in \mathbb{Z}} V_{j}$ is dense in $L^{2}(\mathbb{R})$;

(c) $\bigcap_{j \in \mathbb{Z}} V_{j}=\{0\}$;

(d) $f(x) \in V_{j}$ if and only if $f(2 N x) \in V_{j+1}$ for all $j \in \mathbb{Z}$;

(e) There exists a function $\varphi$ in $V_{0}$ such that $\{\varphi(x-\lambda): \lambda \in \Lambda\}$, is a complete orthonormal basis for $V_{0}$.

It is worth noticing that, when $N=1$, one recovers from the definition above the standard definition of one dimensional multiresolution analysis with dyadic dilation. When, $N>1$, the dilation factor of $2 N$ ensures that $2 N \Lambda \subset \mathbb{Z} \subset \Lambda$.

For every $j \in \mathbb{Z}$, define $W_{j}$ to be the orthogonal complement of $V_{j}$ in $V_{j+1}$. Then we have

$$
V_{j+1}=V_{j} \oplus W_{j} \quad \text { and } \quad W_{\ell} \perp W_{\ell^{\prime}} \quad \text { if } \ell \neq \ell^{\prime} .
$$

It follows that for $j>J$,

$$
V_{j}=V_{J} \oplus \bigoplus_{\ell=0}^{j-J} W_{j-\ell},
$$

where all these subspaces are orthogonal. By virtue of condition (b) in the Definition 2.2, this implies

$$
L^{2}(\mathbb{R})=\bigoplus_{j \in \mathbb{Z}} W_{j}
$$

a decomposition of $L^{2}(\mathbb{R})$ into mutually orthogonal subspaces.

As in the standard case, one expects the existence of $2 N-1$ number of functions so that their translation by elements of $\Lambda$ and dilations by the integral powers of $2 N$ form an orthonormal basis for $L^{2}(\mathbb{R})$. 
Definition 2.3. A set of functions $\left\{\psi_{1}, \psi_{1}, \ldots, \psi_{2 N-1}\right\}$ in $L^{2}(\mathbb{R})$ is said to be a set of basic wavelets associated with the nonuniform multiresolution analysis $\left\{V_{j}: j \in \mathbb{Z}\right\}$ if the family of functions $\left\{\psi_{\ell}(x-\lambda): 1 \leq \ell \leq 2 N-1, \lambda \in \Lambda\right\}$ forms an orthonormal basis for $W_{0}$.

\section{Riesz Bases of Translates}

Lemma 3.1. Let $\varphi, \tilde{\varphi} \in L^{2}(\mathbb{R})$ be given. Then $\{\varphi(x-\lambda): \lambda \in \Lambda\}$ is biorthogonal to $\{\tilde{\varphi}(x-\lambda): \lambda \in$ $\Lambda\}$ if and only if

$$
\sum_{\lambda \in \Lambda} \hat{\varphi}(\xi+\lambda) \overline{\hat{\tilde{\varphi}}(\xi+\lambda)}=1 \quad \text { a.e } \xi \in \mathbb{R}
$$

Proof. For $\lambda, \sigma \in \Lambda$, it follows that $\langle\varphi(x-\lambda), \tilde{\varphi}(x-\sigma)\rangle=\delta_{\lambda, \sigma} \Leftrightarrow\langle\varphi, \tilde{\varphi}(x-\sigma)\rangle=\delta_{0, \sigma}$. Moreover,

$$
\begin{aligned}
\langle\varphi, \tilde{\varphi}(x-\sigma)\rangle & =\langle\hat{\varphi}, \hat{\tilde{\varphi}}(x-\sigma)\rangle \\
& =\int_{\mathbb{R}} \hat{\varphi}(\xi) \overline{\hat{\tilde{\varphi}}(\xi)} e^{-2 \pi i \sigma \xi} d \xi \\
& =\int_{0}^{1 / 2}\left\{\sum_{p \in \mathbb{Z}} \hat{\varphi}\left(\xi+\frac{p}{2}\right) \overline{\hat{\tilde{\varphi}}\left(\xi+\frac{p}{2}\right)} e^{\pi i \sigma p}\right\} e^{-2 \pi i \sigma \xi} d \xi,
\end{aligned}
$$

and using the fact that $\left\{e^{-2 \pi i \sigma \xi}: \sigma \in \Lambda\right\}$ is an orthonormal basis of $L^{2}\left[0, \frac{1}{2}\right)$, we obtain the desired result.

We now provide a sufficient condition for the translates of a function to be linearly independent.

Lemma 3.2. Let $\varphi \in L^{2}(\mathbb{R})$. Suppose there exists two constants $A, B>0$ such that

$$
A \leq \sum_{\lambda \in \Lambda}|\hat{\varphi}(\xi+\lambda)|^{2} \leq B \quad \text { for a.e } \xi \in \mathbb{R} .
$$

Then $\{\varphi(x-\lambda): \lambda \in \Lambda\}$ is linearly independent.

Proof. For the proof of the Lemma, it is sufficient to find another function say $\tilde{\varphi}$ whose translates are biorthogonal to $\varphi$. Let us define the function $\tilde{\varphi}$ by

$$
\hat{\tilde{\varphi}}(\xi)=\frac{\hat{\varphi}(\xi)}{\sum_{\lambda \in \Lambda}|\hat{\varphi}(\xi+\lambda)|^{2}}
$$

By equation (3.1), $\tilde{\varphi}$ is well defined. Now

$$
\sum_{\sigma \in \Lambda} \hat{\varphi}(\xi+\sigma) \overline{\hat{\tilde{\varphi}}(\xi+\sigma)}=\sum_{\sigma \in \Lambda} \hat{\varphi}(\xi+\sigma) \frac{\overline{\hat{\varphi}(\xi+\sigma)}}{\sum_{\lambda \in \Lambda}|\hat{\varphi}(\xi+\lambda+\sigma)|^{2}}
$$




$$
\begin{aligned}
& =\frac{\sum_{\sigma \in \Lambda}|\hat{\varphi}(\xi+\sigma)|^{2}}{\sum_{v \in \Lambda}|\hat{\varphi}(\xi+v)|^{2}} \\
& =1 .
\end{aligned}
$$

Applying Lemma 3.1, it follows that $\{\varphi(x-\lambda): \lambda \in \Lambda\}$ is linearly independent. This completes the proof of the Lemma.

Lemma 3.3. Suppose that the scaling function $\varphi$ satisfies inequality (3.1). Also let $f=\sum_{\lambda \in \Lambda}$ $h_{\lambda} \varphi(x-\lambda)$, where $f \in \operatorname{span}\{\varphi(x-\lambda): \lambda \in \Lambda\}$ and $\left\{h_{\lambda}\right\}$ is a finite sequence. Define the Fourier transform of $h$ by $\hat{h}(\xi)=\sum_{\lambda \in \Lambda} h_{\lambda} e^{-2 \pi i \lambda \xi}$. Then

$$
A \int_{0}^{1 / 2}|\hat{h}(\xi)|^{2} d \xi \leq\|f\|_{2}^{2} \leq B \int_{0}^{1 / 2}|\hat{h}(\xi)|^{2} d \xi .
$$

Proof. Using Plancherel's theorem, we have

$$
\begin{aligned}
\int_{\mathbb{R}}|f(x)|^{2} d x & =\int_{\mathbb{R}}\left|\sum_{\lambda \in \Lambda} h_{\lambda} \varphi(x-\lambda)\right|^{2} d x \\
& =\int_{\mathbb{R}}\left|\sum_{\lambda \in \Lambda} h_{\lambda} \hat{\varphi}(\xi) e^{-2 \pi i \lambda \xi}\right|^{2} d \xi \\
& =\int_{\mathbb{R}}|\hat{\varphi}(\xi)|^{2}\left|\sum_{\lambda \in \Lambda} h_{\lambda} e^{-2 \pi i \lambda \xi}\right|^{2} d \xi \\
& =\int_{\mathbb{R}}|\hat{\varphi}(\xi)|^{2}|\hat{h}(\xi)|^{2} d \xi \\
& =\int_{0}^{1 / 2} \sum_{p \in \mathbb{Z}}\left|\hat{\varphi}\left(\xi+\frac{p}{2}\right)\right|^{2}|\hat{h}(\xi)|^{2} d \xi .
\end{aligned}
$$

Hence, using identity (3.1), we get the desired result.

Theorem 3.4. Let $\{\varphi(x-\lambda): \lambda \in \Lambda\}$ be a Riesz basis for its closed linear span. Assume that there exists a function $\{\tilde{\varphi}(x-\lambda): \lambda \in \Lambda\}$ which is biorthogonal to $\{\varphi(x-\lambda): \lambda \in \Lambda\}$. Then for every $f \in \overline{\operatorname{span}}\{\varphi(x-\lambda): \lambda \in \Lambda\}$, we have

$$
f=\sum_{\lambda \in \Lambda}\langle f, \tilde{\varphi}(x-\lambda)\rangle \varphi(x-\lambda)
$$

and there exists constants $A, B>0$ such that

$$
A\|f\|_{2}^{2} \leq \sum_{\lambda \in \Lambda}|\langle f, \hat{\tilde{\varphi}}(\xi-\lambda)\rangle|^{2} \leq B\|f\|_{2}^{2} .
$$


Proof. We first prove (3.2) and (3.3) for any $f \in \operatorname{span}\{\varphi(x-\lambda): \lambda \in \Lambda\}$ and then generalize it to $\overline{\operatorname{span}}\{\varphi(x-\lambda): \lambda \in \Lambda\}$. Assume that $f \in \operatorname{span}\{\varphi(x-\lambda): \lambda \in \Lambda\}$, then there exists a finite sequence $\left\{h_{\lambda}: \lambda \in \Lambda\right\}$ such that $f=\sum_{\lambda \in \Lambda} h_{\lambda} \varphi(x-\lambda)$. Using biorthogonality, we obtain

$$
\begin{aligned}
\langle f, \tilde{\varphi}(x-\sigma)\rangle & =\left\langle\sum_{\lambda \in \Lambda} h_{\lambda} \varphi(x-\lambda), \tilde{\varphi}(x-\sigma)\right\rangle \\
& =\sum_{\lambda \in \Lambda} h_{\lambda}\langle\varphi(x-\lambda), \tilde{\varphi}(x-\sigma)\rangle \\
& =h_{\lambda} .
\end{aligned}
$$

This proves (3.2). In order to prove (3.3), we make use of Lemma 3.3 to get

$$
B^{-1}\|f\|_{2}^{2} \leq \int_{0}^{1 / 2}|\hat{h}(\xi)|^{2} d \xi \leq A^{-1}\|f\|_{2}^{2} .
$$

Therefore, using the Plancherel formula for Fourier series and the fact that $h_{\lambda}=\langle f, \tilde{\varphi}(x-\lambda)\rangle$, we have

$$
\int_{0}^{1 / 2}|\hat{h}(\xi)|^{2} d \xi=\sum_{\lambda \in \Lambda}\left|h_{\lambda}\right|^{2}=\sum_{\lambda \in \Lambda}|\langle f, \tilde{\varphi}(x-\lambda)\rangle|^{2} .
$$

This proves (3.3). We now generalize the results to $\overline{\operatorname{span}}\{\varphi(x-\lambda): \lambda \in \Lambda\}$. Let us first prove (3.3). For $f \in \overline{\operatorname{span}}\{\tilde{\varphi}(x-\lambda): \lambda \in \Lambda\}$, there exists a sequence $\left\{f_{m}: m \in \mathbb{Z}\right\}$ in $\operatorname{span}\{\tilde{\varphi}(x-\lambda): \lambda \in$ $\Lambda$ \} such that $\left\|f_{m}-f\right\|_{2} \rightarrow 0$ as $m \rightarrow \infty$. Thus for each $\lambda \in \Lambda$, we have

$$
\left\langle f_{m}, \tilde{\varphi}(x-\lambda)\right\rangle \rightarrow\langle f, \tilde{\varphi}(x-\lambda)\rangle \quad \text { as } m \rightarrow \infty
$$

So the result holds for each $f_{m}$. Therefore,

$$
\begin{aligned}
\sum_{\lambda \in \Lambda}|\langle f, \tilde{\varphi}(x-\lambda)\rangle|^{2} & =\sum_{\lambda \in \Lambda} \lim _{m \rightarrow \infty}\left|\left\langle f_{m}, \tilde{\varphi}(x-\lambda)\right\rangle\right|^{2} \\
& =\lim _{m \rightarrow \infty} \sum_{\lambda \in \Lambda}\left|\left\langle f_{m}, \tilde{\varphi}(x-\lambda)\right\rangle\right|^{2} \\
& \leq B \lim _{m \rightarrow \infty}\left\|f_{m}\right\|_{2}^{2} \\
& =B\|f\|_{2}^{2} .
\end{aligned}
$$

Thus, the upper bound holds in (3.3). Further, we have

$$
\left\{\sum_{\lambda \in \Lambda}\left|\left\langle f_{m}, \tilde{\varphi}(x-\lambda)\right\rangle\right|^{2}\right\}^{1 / 2} \leq\left\{\sum_{\lambda \in \Lambda}\left|\left\langle f_{m}-f, \tilde{\varphi}(x-\lambda)\right\rangle\right|^{2}\right\}^{1 / 2}+\left\{\sum_{\lambda \in \Lambda}|\langle f, \tilde{\varphi}(x-\lambda)\rangle|^{2}\right\}^{1 / 2} .
$$

As the upper bound in (3.3) holds for $f_{m}-f$ and lower bound for each $f_{m}$, we get

$$
A^{1 / 2}\left\|f_{m}\right\|_{2} \leq B^{1 / 2}\left\|f_{m}-f\right\|_{2}+\left(\sum_{\lambda \in \Lambda}\left|\left\langle f_{m}, \tilde{\varphi}(x-\lambda)\right\rangle\right|^{2}\right)^{1 / 2}
$$


from which, it follows that

$$
A\|f\|_{2}^{2} \leq \sum_{\lambda \in \Lambda}|\langle f, \tilde{\varphi}(x-\lambda)\rangle|^{2}
$$

This proves (3.3). Similarly, we can prove (3.2) for $f \in \overline{\operatorname{span}}\{\varphi(x-\lambda): \lambda \in \Lambda\}$ and the proof of the theorem is complete.

\section{Biorthogonal Properties of Nonuniform Wavelets}

Let $\left\{V_{j}: j \in \mathbb{Z}\right\}$ and $\left\{\tilde{V}_{j}: j \in \mathbb{Z}\right\}$ be biorthogonal NUMRA's with scaling functions $\varphi$ and $\tilde{\varphi}$. Then there exists integral periodic functions $m_{0}$ and $\tilde{m}_{0}$ such that $\hat{\varphi}(\xi)=m_{0}(\xi / 2 N) \hat{\varphi}(\xi / 2 N)$ and $\hat{\tilde{\varphi}}(\xi)=\tilde{m}_{0}(\xi / 2 N) \hat{\tilde{\varphi}}(\xi / 2 N)$. Suppose there exists integral periodic functions $m_{\ell}$ and $\tilde{m}_{\ell}$, $1 \leq \ell \leq 2 N-1$ such that

$$
\mathscr{M}(\xi) \overline{\tilde{M}(\xi)}=I
$$

where

$$
\mathscr{M}(\xi)=\left(\begin{array}{cccc}
m_{0}\left(\frac{\xi}{2 N}\right) & m_{0}\left(\frac{\xi}{2 N}+\frac{1}{4 N}\right) & \ldots & m_{0}\left(\frac{\xi}{2 N}+\frac{2 N-1}{4 N}\right) \\
m_{1}\left(\frac{\xi}{2 N}\right) & m_{1}\left(\frac{\xi}{2 N}+\frac{1}{4 N}\right) & \ldots & m_{1}\left(\frac{\xi}{2 N}+\frac{2 N-1}{4 N}\right) \\
\vdots & \vdots & \ddots & \vdots \\
m_{2 N-1}\left(\frac{\xi}{2 N}\right) & m_{2 N-1}\left(\frac{\xi}{2 N}+\frac{1}{4 N}\right) & \ldots & m_{2 N-1}\left(\frac{\xi}{2 N}+\frac{2 N-1}{4 N}\right)
\end{array}\right)
$$

and

$$
\tilde{\tilde{M}}(\xi)=\left(\begin{array}{cccc}
\tilde{m}_{0}\left(\frac{\xi}{2 N}\right) & \tilde{m}_{0}\left(\frac{\xi}{2 N}+\frac{1}{4 N}\right) & \ldots & \tilde{m}_{0}\left(\frac{\xi}{2 N}+\frac{2 N-1}{4 N}\right) \\
\tilde{m}_{1}\left(\frac{\xi}{2 N}\right) & \tilde{m}_{1}\left(\frac{\xi}{2 N}+\frac{1}{4 N}\right) & \ldots & \tilde{m}_{1}\left(\frac{\xi}{2 N}+\frac{2 N-1}{4 N}\right) \\
\vdots & \vdots & \ddots & \vdots \\
\tilde{m}_{2 N-1}\left(\frac{\xi}{2 N}\right) & \tilde{m}_{2 N-1}\left(\frac{\xi}{2 N}+\frac{1}{4 N}\right) & \ldots & \tilde{m}_{2 N-1}\left(\frac{\xi}{2 N}+\frac{2 N-1}{4 N}\right)
\end{array}\right) .
$$

For $1 \leq \ell \leq 2 N-1$, define the associated wavelets as $\psi_{\ell}$ and $\tilde{\psi}_{\ell}$ by

$$
\hat{\psi}_{\ell}(\xi)=m_{\ell}(\xi / 2 N) \hat{\varphi}(\xi / 2 N) \quad \text { and } \quad \hat{\tilde{\psi}}_{\ell}(\xi)=\tilde{m}_{\ell}(\xi / 2 N) \hat{\tilde{\varphi}}(\xi / 2 N)
$$

Definition 4.1. A pair of NUMRA's $\left\{V_{j}: j \in \mathbb{Z}\right\}$ and $\left\{\tilde{V}_{j}: j \in \mathbb{Z}\right\}$ with scaling functions $\varphi$ and $\tilde{\varphi}$ respectively are said to be dual to each other if $\{\varphi(\cdot-\lambda): \lambda \in \Lambda\}$ and $\{\tilde{\varphi}(\cdot-\lambda): \lambda \in \Lambda\}$ are biorthogonal.

Definition 4.2. Let $\varphi$ and $\tilde{\varphi}$ be scaling functions for dual NUMRA's. For each $j \in \mathbb{Z}$, define the operators $P_{j}$ and $\tilde{P}_{j}$ on $L^{2}(\mathbb{R})$ by

$$
P_{j} f=\sum_{\lambda \in \Lambda}\left\langle f, \tilde{\varphi}_{j, \lambda}\right\rangle \varphi_{j, \lambda} \quad \text { and } \quad \tilde{P}_{j} f=\sum_{\lambda \in \Lambda}\left\langle f, \varphi_{j, \lambda}\right\rangle \tilde{\varphi}_{j, \lambda}
$$


respectively. Here $\varphi_{j, \lambda}=\delta_{j} \varphi(\cdot-\lambda)$ and $\tilde{\varphi}_{j, \lambda}=\delta_{j} \tilde{\varphi}(\cdot-\lambda)$. Same is the case with $\psi_{j, \lambda}$ and $\tilde{\psi}_{j, \lambda}$. It is easy to verify that these operators are uniformly bounded on $L^{2}(\mathbb{R})$ and both the series are convergent in $L^{2}(\mathbb{R})$.

Remark 4.3. The operators $P_{j}$ and $\tilde{P}_{j}$ satisfy the following properties.

(a) $P_{j} f=f$ if and only if $f \in V_{j}$ and $\tilde{P}_{j} f=f$ if and only if $f \in \tilde{V}_{j}$.

(b) $\lim _{j \rightarrow \infty}\left\|P_{j} f-f\right\|_{2}=0$ and $\lim _{j \rightarrow-\infty}\left\|P_{j} f\right\|_{2}=0$ for every $f \in L^{2}(\mathbb{R})$.

Theorem 4.4. Let $\varphi$ and $\tilde{\varphi}$ be the scaling functions for dual NUMRA's and $\psi_{\ell}$ and $\tilde{\psi}_{\ell}, 1 \leq \ell \leq$ $2 N-1$ be the associated wavelets satisfying (4.1). Then, we have the following

(a) $\left\{\psi_{\ell, 0, \lambda}: \lambda \in \Lambda\right\}$ is biorthogonal to $\left\{\tilde{\psi}_{\ell, 0, \sigma}: \sigma \in \Lambda\right\}$,

(b) $\left\langle\psi_{\ell, 0, \lambda}, \tilde{\varphi}_{0, \sigma}\right\rangle=\left\langle\tilde{\psi}_{\ell, 0, \lambda}, \varphi_{0, \sigma}\right\rangle$, for all $\lambda, \sigma \in \Lambda$.

Proof. we have

$$
\begin{aligned}
& \sum_{p \in \mathbb{Z}} \hat{\psi}_{\ell}\left(\xi+\frac{p}{2}\right) \overline{\hat{\tilde{\psi}}_{\ell}\left(\xi+\frac{p}{2}\right)} \\
& =\sum_{p \in \mathbb{Z}}\left\{m_{\ell}\left(\frac{\xi}{2 N}+\frac{p}{4 N}\right) \hat{\varphi}\left(\frac{\xi}{2 N}+\frac{p}{4 N}\right) \overline{\tilde{m}_{\ell}\left(\frac{\xi}{2 N}+\frac{p}{4 N}\right) \overline{\hat{\varphi}\left(\frac{\xi}{2 N}+\frac{p}{4 N}\right)}}\right\} \\
& =\sum_{s=0}^{2 N-1} \sum_{p \in \mathbb{Z}}\left\{m_{\ell}\left(\frac{\xi}{2 N}+\frac{p}{2}+\frac{s}{4 N}\right) \hat{\varphi}\left(\frac{\xi}{2 N}+\frac{p}{2}+\frac{s}{4 N}\right) \overline{\tilde{m}_{\ell}\left(\frac{\xi}{2 N}+\frac{p}{2}+\frac{s}{4 N}\right)} \hat{\hat{\varphi}\left(\frac{\xi}{2 N}+\frac{p}{2}+\frac{s}{4 N}\right)}\right\} \\
& =\sum_{s=0}^{2 N-1}\left\{m_{\ell}\left(\frac{\xi}{2 N}+\frac{s}{4 N}\right) \overline{\tilde{m}_{\ell}\left(\frac{\xi}{2 N}+\frac{s}{4 N}\right)}\right\} \\
& =1 .
\end{aligned}
$$

Hence, by Lemma 3.1, $\left\{\psi_{\ell, 0, \lambda}: \lambda \in \Lambda\right\}$ is biorthogonal to $\left\{\tilde{\psi}_{\ell, 0, \lambda}: \lambda \in \Lambda\right\}$. This proves part (a). To prove part (b), we have for, $\lambda, \sigma \in \Lambda$

$$
\begin{aligned}
& \left\langle\psi_{\ell, 0, \lambda}, \tilde{\varphi}_{0, \sigma}\right\rangle=\left\langle\psi_{\ell}(x-\lambda), \tilde{\varphi}(x-\sigma)\right\rangle \\
& =\left\langle\hat{\psi}_{\ell} e^{-2 \pi i \lambda}, \hat{\tilde{\varphi}} e^{-2 \pi i \sigma}\right\rangle \\
& =\int_{\mathbb{R}} m_{\ell}\left(\frac{\xi}{2 N}\right) \hat{\varphi}\left(\frac{\xi}{2 N}\right) e^{-2 \pi i \lambda \xi} \overline{\tilde{m}_{0}\left(\frac{\xi}{2 N}\right)} \overline{\hat{\varphi}\left(\frac{\xi}{2 N}\right)} e^{2 \pi i \sigma \xi} d \xi \\
& =\int_{0}^{1 / 2} \sum_{p \in \mathbb{Z}}\left\{m_{\ell}\left(\frac{\xi}{2 N}+\frac{p}{4 N}\right) \hat{\varphi}\left(\frac{\xi}{2 N}+\frac{p}{4 N}\right)\right.
\end{aligned}
$$

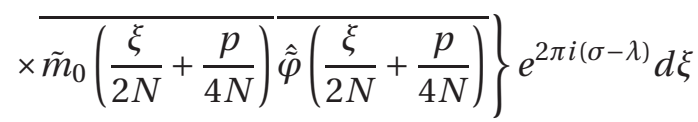

$$
\begin{aligned}
& =\int_{0}^{1 / 2} \sum_{s=0}^{2 N-1} \sum_{p \in \mathbb{Z}}\left\{m_{\ell}\left(\frac{\xi}{2 N}+\frac{p}{2}+\frac{s}{4 N}\right) \hat{\varphi}\left(\frac{\xi}{2 N}+\frac{p}{2}+\frac{s}{4 N}\right)\right.
\end{aligned}
$$




$$
\begin{aligned}
& \left.\times \overline{m_{0}\left(\frac{\xi}{2 N}+\frac{p}{2}+\frac{s}{4 N}\right)} \overline{\hat{\tilde{\varphi}}\left(\frac{\xi}{2 N}+\frac{p}{2}+\frac{s}{4 N}\right)}\right\} e^{2 \pi i(\sigma-\lambda)} d \xi \\
= & \int_{0}^{1 / 2} \sum_{s=0}^{2 N-1}\left\{m_{\ell}\left(\frac{\xi}{2 N}+\frac{s}{4 N}\right) \overline{\tilde{m}_{0}\left(\frac{\xi}{2 N}+\frac{s}{4 N}\right)}\right\} e^{2 \pi i(\sigma-\lambda)} d \xi \\
= & 0 .
\end{aligned}
$$

The dual one can also be shown equal to zero in a similar manner. This proves part (b) and hence completes the proof of the theorem.

Theorem 4.5. Let $\varphi$ and $\tilde{\varphi}$ and $\psi_{\ell}$ and $\tilde{\psi}_{\ell}, 1 \leq \ell \leq 2 N-1$ be as in Theorem 4.4. Let $\psi_{0}=\varphi$ and $\tilde{\psi}_{0}=\tilde{\varphi}$. Then for every $f \in L^{2}(\mathbb{R})$, we have

$$
P_{1} f=P_{0} f+\sum_{\ell=1}^{2 N-1} \sum_{\lambda \in \Lambda}\left\langle f, \tilde{\psi}_{\ell, 0, \lambda}\right\rangle \psi_{\ell, 0, \lambda}
$$

and

$$
\tilde{P}_{1} f=\tilde{P}_{0} f+\sum_{\ell=1}^{2 N-1} \sum_{\lambda \in \Lambda}\left\langle f, \psi_{\ell, 0, \lambda}\right\rangle \tilde{\psi}_{\ell, 0, \lambda} .
$$

where the series in (4.2) and (4.3) converges in $L^{2}(\mathbb{R})$.

Proof. For the sake of convenience, we will only prove (4.2), as (4.3) is an easy consequence. In particular, we will prove it in the weak sense only. For this, let $f, g \in L^{2}(\mathbb{R})$. Then, we have

$$
\begin{aligned}
& \sum_{\ell=0}^{2 N-1} \sum_{\lambda \in \Lambda}\left\langle f, \tilde{\psi}_{\ell, 0, \lambda}\right\rangle \overline{\left\langle g, \psi_{\ell, 0, \lambda}\right\rangle} \\
& =\sum_{\ell=0}^{2 N-1} \sum_{\lambda \in \Lambda}\left\{\int_{\mathbb{R}} \hat{f}(\xi) \overline{\hat{\tilde{\psi}}_{\ell}(\xi)} e^{2 \pi i \lambda \xi} d \xi\right\}\left\{\int_{\mathbb{R}} \overline{\hat{g}(\xi)} \hat{\psi}_{\ell}(\xi) e^{-2 \pi i \lambda \xi} d \xi\right\} \\
& =\sum_{\ell=0}^{2 N-1} \sum_{\lambda \in \Lambda}\left\{\int_{0}^{1 / 2} \sum_{p \in \mathbb{Z}} \hat{f}\left(\xi+\frac{p}{2}\right) \overline{\hat{\tilde{\psi}} \ell\left(\xi+\frac{p}{2}\right)} e^{2 \pi i \lambda \xi} d \xi\right\} \\
& \times\left\{\int_{0}^{1 / 2} \sum_{q \in \mathbb{Z}} \overline{\hat{g}\left(\xi+\frac{q}{2}\right)} \hat{\psi} \ell\left(\xi+\frac{q}{2}\right) e^{-2 \pi i \lambda \xi} d \xi\right\} \\
& =\sum_{\ell=0}^{2 N-1} \int_{0}^{1 / 2}\left\{\sum_{p \in \mathbb{Z}} \hat{f}\left(\xi+\frac{p}{2}\right) \overline{\hat{\tilde{\psi}}_{\ell}\left(\xi+\frac{p}{2}\right)}\right\}\left\{\sum_{q \in \mathbb{Z}} \overline{\hat{g}\left(\xi+\frac{q}{2}\right)} \hat{\psi}_{\ell}\left(\xi+\frac{q}{2}\right)\right\} d \xi \\
& =\int_{0}^{1 / 2} \sum_{\ell=0}^{2 N-1}\left\{\sum_{p \in \mathbb{Z}} \hat{f}\left(\xi+\frac{p}{2}\right) \overline{\tilde{m}_{\ell}\left(\frac{\xi}{2 N}+\frac{p}{4 N}\right)} \overline{\tilde{\varphi}\left(\frac{\xi}{2 N}+\frac{p}{4 N}\right)}\right. \\
& \left.\times \sum_{q \in \mathbb{Z}} \overline{\hat{g}\left(\xi+\frac{q}{2}\right)} m_{\ell}\left(\frac{\xi}{2 N}+\frac{q}{4 N}\right) \hat{\varphi}\left(\frac{\xi}{2 N}+\frac{q}{4 N}\right)\right\} d \xi \\
& =\int_{0}^{1 / 2} \sum_{\ell=0}^{2 N-1}\left\{\sum_{r=0}^{2 N-1} \sum_{p^{\prime} \in \mathbb{Z}} \hat{f}\left(\xi+\frac{p^{\prime}}{2} N+\frac{r}{2}\right) \overline{\tilde{m}_{\ell}\left(\frac{\xi}{2 N}+\frac{r}{4 N}+\frac{p^{\prime}}{2}\right)} \hat{\tilde{\varphi}\left(\frac{\xi}{2 N}+\frac{r}{4 N}+\frac{p^{\prime}}{2}\right)}\right.
\end{aligned}
$$




$$
\begin{aligned}
& \left.\times \sum_{s=0}^{2 N-1} \sum_{q^{\prime} \in \mathbb{N}_{0}} \overline{g\left(\xi+\frac{q^{\prime}}{2} N+\frac{s}{2}\right)} m_{\ell}\left(\frac{\xi}{2 N}+\frac{s}{4 N}+\frac{q^{\prime}}{2}\right) \hat{\varphi}\left(\frac{\xi}{2 N}+\frac{s}{4 N}+\frac{q^{\prime}}{2}\right)\right\} d \xi \\
& =\int_{0}^{1 / 2} \sum_{r=0}^{2 N-1} \sum_{p^{\prime} \in \mathbb{Z}}^{2 N-1} \sum_{s=0}^{2} \sum_{q^{\prime} \in \mathbb{N}_{0}}\left\{\sum_{\ell=0}^{2 N-1} \overline{m_{\ell}\left(\frac{\xi}{2 N}+\frac{r}{4 N}\right)} m_{\ell}\left(\frac{\xi}{2 N}+\frac{s}{4 N}\right)\right\} \\
& \times \hat{f}\left(\xi+\frac{p^{\prime}}{2} N+\frac{r}{2}\right) \overline{\hat{\tilde{\varphi}}\left(\frac{\xi}{2 N}+\frac{r}{4 N}+\frac{p^{\prime}}{2}\right)} \hat{\hat{g}\left(\xi+\frac{q^{\prime}}{2} N+\frac{s}{2}\right)} \hat{\varphi}\left(\frac{\xi}{2 N}+\frac{s}{4 N}+\frac{q^{\prime}}{2}\right) d \xi \\
& =\int_{0}^{1 / 2} \sum_{p^{\prime} \in \mathbb{Z}} \sum_{q^{\prime} \in \mathbb{N}_{0}} \sum_{s=0}^{2 N-1} \hat{f}\left(\xi+\frac{p^{\prime}}{2} N+\frac{s}{2}\right) \overline{\hat{\tilde{\varphi}}\left(\frac{\xi}{2 N}+\frac{s}{4 N}+\frac{p^{\prime}}{2}\right)} \\
& \times \bar{g}\left(\xi+\frac{q^{\prime}}{2} N+\frac{s}{2}\right) \hat{\varphi}\left(\frac{\xi}{2 N}+\frac{s}{4 N}+\frac{p^{\prime}}{2}\right) d \xi \\
& =\sum_{s=0}^{2 N-1} \int_{0}^{s+1 / 2} \sum_{p^{\prime} \in \mathbb{Z}} \sum_{q^{\prime} \in \mathbb{N}_{0}} \hat{f}\left(\xi+\frac{p^{\prime}}{2} N\right) \overline{\hat{\tilde{\varphi}}\left(\frac{\xi}{2 N}+\frac{p^{\prime}}{2}\right) \bar{g}\left(\xi+\frac{q^{\prime}}{2} N\right)} \hat{\varphi}\left(\frac{\xi}{2 N}+\frac{p^{\prime}}{2}\right) d \xi .
\end{aligned}
$$

Moreover, we have

$$
\begin{aligned}
& \sum_{\lambda \in \Lambda}\left\langle f, \tilde{\varphi}_{1, \lambda}\right\rangle \overline{\left\langle g, \varphi_{1, \lambda}\right\rangle}=\sum_{\lambda \in \Lambda}\left\{\int_{\mathbb{R}} \hat{f}(\xi) \overline{\hat{\varphi}\left(\frac{\xi}{2 N}\right)} e^{2 \pi i \xi / 2 N} d \xi\right\}\left\{\int_{\mathbb{R}} \overline{\hat{g}(\xi)} \hat{\varphi}\left(\frac{\xi}{2 N}\right) e^{-2 \pi i \xi / 2 N} d \xi\right\} \\
& =\int_{0}^{1 / 2} \sum_{p \in \mathbb{Z}} \hat{f}\left(\xi+\frac{p}{2} N\right) \overline{\hat{\tilde{\varphi}}\left(\frac{\xi}{2 N}+\frac{p}{2}\right)} d \xi \int_{0}^{1 / 2} \sum_{q \in \mathbb{Z}} \overline{\hat{g}\left(\xi+\frac{q}{2} N\right)} \hat{\varphi}\left(\frac{\xi}{2 N}+\frac{q}{2}\right) d \xi \\
& =\int_{0}^{1 / 2} \sum_{p \in \mathbb{Z}} \hat{f}\left(\xi+\frac{p}{2} N\right) \overline{\hat{\tilde{\varphi}}\left(\frac{\xi}{2 N}+\frac{p}{2}\right)} d \xi \int_{0}^{1 / 2} \sum_{q \in \mathbb{Z}} \overline{\hat{g}\left(\xi+\frac{q}{2} N\right)} \hat{\varphi}\left(\frac{\xi}{2 N}+\frac{q}{2}\right) d \xi \\
& =\int_{0}^{1 / 2} \sum_{p \in \mathbb{Z}} \sum_{q \in \mathbb{Z}} \hat{f}\left(\xi+\frac{p}{2} N\right) \overline{\hat{\tilde{\varphi}}\left(\frac{\xi}{2 N}+\frac{p}{2}\right)} \overline{\hat{g}\left(\xi+\frac{q}{2} N\right)} \hat{\varphi}\left(\frac{\xi}{2 N}+\frac{q}{2}\right) d \xi .
\end{aligned}
$$

Combing (4.4) and (4.5), we get the desired result.

Theorem 4.6. Let $\varphi$ and $\tilde{\varphi}$ and $\psi_{\ell}$ and $\tilde{\psi} \ell, 1 \leq \ell \leq 2 N-1$ be as in Theorem ??. Then, for every $f \in L^{2}(\mathbb{R})$, we have

$$
f=\sum_{\ell=1}^{2 N-1} \sum_{j \in \mathbb{Z}} \sum_{\lambda \in \Lambda}\left\langle f, \tilde{\psi}_{\ell, j, \lambda}\right\rangle \psi_{\ell, j, \lambda}=\sum_{\ell=1}^{2 N-1} \sum_{j \in \mathbb{Z}} \sum_{\lambda \in \Lambda}\left\langle f, \psi_{\ell, j, \lambda}\right\rangle_{\ell} \tilde{\psi}_{\ell, j, \lambda},
$$

where the series converges in $L^{2}(\mathbb{R})$.

Proof. Using Remark 4.3 and Theorem 4.5, proof of Theorem 4.6 follows.

Theorem 4.7. Let $\varphi$ and $\tilde{\varphi}$ be the scaling functions for dual NUMRA's and $\psi_{\ell}$ and $\tilde{\psi}_{\ell}, 1 \leq \ell \leq$ $2 N-1$ be the associated wavelets satisfying the matrix condition (4.1). Then, the collection $\left\{\psi_{\ell, j, \lambda}: 1 \leq \ell \leq 2 N-1, j \in \mathbb{Z}, \lambda \in \Lambda\right\}$ and $\left\{\tilde{\psi}_{\ell, j, \lambda}: 1 \leq \ell \leq 2 N-1, j \in \mathbb{Z}, \lambda \in \Lambda\right\}$ are biorthogonal. Moreover, if

$$
|\hat{\varphi}(\xi)| \leq C(1+|\xi|)^{-\frac{1}{2}-\epsilon},|\hat{\tilde{\varphi}}(\xi)| \leq C(1+|\xi|)^{-\frac{1}{2}-\epsilon},\left|\hat{\psi}_{\ell}(\xi)\right| \leq C|\xi| \text { and }|\hat{\tilde{\psi}}(\xi)| \leq C|\xi|,
$$


for some constant $C>0, \epsilon>0$ and for a.e. $\xi \in \mathbb{R}$, then $\left\{\psi_{\ell, j, \lambda}: 1 \leq \ell \leq 2 N-1, j \in \mathbb{Z}, \lambda \in \Lambda\right\}$ and $\left\{\tilde{\psi}_{\ell, j, \lambda}: 1 \leq \ell \leq 2 N-1, j \in \mathbb{Z}, \lambda \in \Lambda\right\}$ form Riesz bases for $L^{2}(\mathbb{R})$.

Proof. First we show that $\left\{\psi_{\ell, j, \lambda}: 1 \leq \ell \leq 2 N-1, j \in \mathbb{Z}, \lambda \in \Lambda\right\}$ and $\left\{\tilde{\psi}_{\ell, j, \lambda}: 1 \leq \ell \leq 2 N-1, j \in\right.$ $\mathbb{Z}, \lambda \in \Lambda\}$ are biorthogonal to each other. For this, we will show that for each $\ell, 1 \leq \ell \leq 2 N-1$ and $j \in \mathbb{Z}$,

$$
\left\langle\psi_{\ell, j, \lambda}, \tilde{\psi}_{\ell, j, \sigma}\right\rangle=\delta_{\lambda, \sigma} .
$$

We have in fact already proved (4.8) for $j=0$. For $j \neq 0$, we have

$$
\left\langle\psi_{\ell, j, \lambda}, \tilde{\psi}_{\ell, j, \sigma}\right\rangle=\left\langle P_{-j} \psi_{\ell, 0, \lambda}, P_{-j} \tilde{\psi}_{\ell, 0, \sigma}\right\rangle=\left\langle\psi_{\ell, 0, \lambda}, \tilde{\psi}_{\ell, 0, \sigma}\right\rangle=\delta_{\lambda, \sigma} .
$$

For fixed $\lambda, \sigma \in \Lambda$ and $j, j^{\prime} \in \mathbb{Z}$ with $j<j^{\prime}$, we claim that

$$
\left\langle\psi_{\ell, j, \lambda}, \tilde{\psi}_{\ell^{\prime}, j^{\prime}, \sigma}\right\rangle=0
$$

As $\psi_{\ell, 0, \lambda} \in V_{1}$, hence $\psi_{\ell, j, \lambda}=P_{-j} \psi_{\ell, 0, \lambda} \in V_{j+1} \subseteq V_{j^{\prime}}$. Therefore, it is enough to show that $\tilde{\psi} \ell^{\prime}, j^{\prime}, \sigma$ is orthogonal to every element of $V_{j^{\prime}}$. Let $f \in V_{j^{\prime}}$. Since $\left\{\varphi_{j^{\prime}, \lambda}: \lambda \in \Lambda\right\}$ is a Riesz basis for $V_{j^{\prime}}$, hence there exists an $l^{2}$-sequence $\left\{d_{\lambda}: \lambda \in \Lambda\right\}$ such that $f=\sum_{\lambda \in \Lambda} d_{\lambda} \varphi_{j^{\prime}, \lambda}$ in $L^{2}(\mathbb{R})$. Using part (b) of Lemma 4.1, we have

$$
\left\langle\tilde{\psi}_{\ell^{\prime}, j^{\prime}, \sigma}, \varphi_{j^{\prime}, \lambda}\right\rangle=\left\langle P_{-j^{\prime}} \tilde{\psi}_{\ell^{\prime}, 0, \sigma}, P_{-j^{\prime}} \varphi_{0, \lambda}\right\rangle=0 .
$$

Therefore,

$$
\left\langle\tilde{\psi}_{\ell^{\prime}, j^{\prime}, \sigma}, f\right\rangle=\left\langle\tilde{\psi}_{\ell^{\prime}, j^{\prime}, \sigma}, \sum_{\lambda \in \Lambda} d_{\lambda} \varphi_{j^{\prime}, \lambda}\right\rangle=\sum_{\lambda \in \Lambda} d_{\lambda}\left\langle\tilde{\psi}_{\ell^{\prime}, j^{\prime}, \sigma}, \varphi_{j^{\prime}, \lambda}\right\rangle=0 .
$$

We now show that these two collections form Riesz bases for $L^{2}(\mathbb{R})$. Linear independence is clear from the fact that these collections are biorthogonal to each other. So, we have to check the frame inequalities i.e., there must exist constants $A, \tilde{A}, B, \tilde{B}>0$ such that

$$
A\|f\|_{2}^{2} \leq \sum_{\ell=1}^{2 N-1} \sum_{j \in \mathbb{Z}} \sum_{\lambda \in \Lambda}\left|\left\langle f, \psi_{\ell, j, \lambda}\right\rangle\right|^{2} \leq B\|f\|_{2}^{2}, \quad \text { for all } f \in L^{2}(\mathbb{R}),
$$

and

$$
\tilde{A}\|f\|_{2}^{2} \leq \sum_{\ell=1}^{2 N-1} \sum_{j \in \mathbb{Z}} \sum_{\lambda \in \Lambda}\left|\left\langle f, \tilde{\psi}_{\ell, j, \lambda}\right\rangle\right|^{2} \leq \tilde{B}\|f\|_{2}^{2}, \quad \text { for all } f \in L^{2}(\mathbb{R}) .
$$

Let us first check the existence of the upper bounds in (4.10) and (4.11). For this, we have

$$
\begin{aligned}
\sum_{\lambda \in \Lambda}\left|\left\langle f, \tilde{\psi}_{\ell, j, \lambda}\right\rangle\right|^{2} & =\sum_{\lambda \in \Lambda}\left|\int_{\mathbb{R}} \hat{f}(\xi)(2 N)^{-j / 2} \overline{\hat{\psi}_{\ell}\left((2 N)^{-j} \xi\right)} e^{2 \pi i \lambda(2 N)^{-j} \xi} d \xi\right|^{2} \\
& =(2 N)^{-j} \sum_{\lambda \in \Lambda}\left|\int_{0}^{1 / 2} \sum_{p \in \mathbb{Z}} \hat{f}\left(\xi+(2 N)^{j} \frac{p}{2}\right) \overline{\hat{\psi}_{\ell}\left((2 N)^{-j} \xi+\frac{p}{2}\right)} e^{2 \pi i \lambda(2 N)^{-j} \xi} d \xi\right|^{2}
\end{aligned}
$$




$$
\begin{aligned}
& =\int_{0}^{1 / 2}\left|\sum_{p \in \mathbb{Z}} \hat{f}\left(\xi+(2 N)^{j} \frac{p}{2}\right) \overline{\hat{\psi}_{\ell}\left((2 N)^{-j} \xi+\frac{p}{2}\right)}\right|^{2} d \xi \\
& =\int_{0}^{1 / 2}\left\{\sum_{p \in \mathbb{Z}}\left|\hat{f}\left(\xi+(2 N)^{j} \frac{p}{2}\right)\right|^{2}\left|\hat{\psi}_{\ell}\left((2 N)^{-j} \xi+\frac{p}{2}\right)\right|^{2 \delta}\right\} \\
& \times\left\{\sum_{q \in \mathbb{Z}}\left|\hat{\psi}_{\ell}\left((2 N)^{-j} \xi+\frac{q}{2}\right)\right|^{2(1-\delta)}\right\} d \xi \\
& =\int_{\mathbb{R}}|\hat{f}(\xi)|^{2}\left|\hat{\psi}_{\ell}\left((2 N)^{-j} \xi\right)\right|^{2 \delta} \sum_{q \in \mathbb{Z}}\left|\hat{\psi}_{\ell}\left((2 N)^{-j} \xi+\frac{q}{2}\right)\right|^{2(1-\delta)} d \xi .
\end{aligned}
$$

By our assumption (4.7), we have $\left|\hat{\psi}_{\ell}(\xi)\right| \leq C\left(1+\left|(2 N)^{-1} \xi\right|\right)^{-1 / 2-\epsilon}$ and thereforeit follows that $\sum_{q \in \mathbb{Z}}\left|\hat{\psi}_{\ell}\left((2 N)^{-j} \xi+q / 2\right)\right|^{2(1-\delta)}$ is uniformly bounded if $\delta<2 \epsilon(1+2 \epsilon)^{-1}$. Thus, there exists a constant $C>0$ such that

$$
\begin{aligned}
\sum_{\lambda \in \Lambda}\left|\left\langle f, \tilde{\psi}_{\ell, j, \lambda}\right\rangle\right|^{2} & \leq C \int_{\mathbb{R}}|\hat{f}(\xi)|^{2} \sum_{j \in \mathbb{Z}}\left|\hat{\psi}_{\ell}\left((2 N)^{-j} \xi\right)\right|^{2 \delta} d \xi \\
& \leq C \sup \left\{\sum_{j \in \mathbb{Z}}\left|\hat{\psi}_{\ell}\left((2 N)^{-j} \xi\right)\right|^{2 \delta}: \xi \in[1,2 N]\right\}\|f\|_{2}^{2} .
\end{aligned}
$$

Moreover for $\xi \in[1,2 N]$, we have

$$
\begin{aligned}
\sum_{j=-\infty}^{0}\left|\hat{\psi}_{\ell}\left((2 N)^{-j} \xi\right)\right|^{2 \delta} & \leq \sum_{j=-\infty}^{0} \frac{C^{2 \delta}}{\left(1+\left|(2 N)^{j-1} \xi\right|\right)^{\delta(1+2 \epsilon)}} \leq \sum_{j=-\infty}^{0} \frac{C^{2 \delta}}{(2 N)^{(j-1) \delta(1+2 \epsilon)}} \\
& \leq C^{2 \delta} \frac{q^{\delta(1+2 \epsilon)}}{1-(2 N)^{-\delta(1+2 \epsilon)}} .
\end{aligned}
$$

Furthermore,

$$
\sum_{j=1}^{\infty}\left|\hat{\psi}_{\ell}\left((2 N)^{-j} \xi\right)\right|^{2 \delta} \leq \sum_{j=1}^{\infty}\left(C(2 N)^{-j}|\xi|\right)^{2 \delta} \leq C^{2 \delta} \sum_{j=1}^{\infty}(2 N)^{(-j+1) 2 \delta}=C^{2 \delta} \frac{1}{1-(2 N)^{-2 \delta}} .
$$

Therefore, it follows that $\sup \left\{\sum_{j \in \mathbb{Z}}\left|\hat{\psi}_{\ell}\left((2 N)^{-j} \xi\right)\right|^{2 \delta}: \xi \in[1,2 N]\right\}$ is finite. Hence, there exist $B>0$ such that of (4.10) holds. Similarly, we can show for dual one also. The existence of lower bounds for both the cases can be shown in similar fashion. Using Theorem 4.6, it follows that if $f \in L^{2}(\mathbb{R})$, then (4.6) holds. Thus, we have

$$
\begin{aligned}
\|f\|_{2}^{2} & =\langle f, f\rangle \\
& =\left\langle\sum_{\ell=1}^{2 N-1} \sum_{j \in \mathbb{Z}} \sum_{\lambda \in \Lambda}\left\langle f, \tilde{\psi}_{\ell, j, \lambda}\right\rangle_{\ell, j, \lambda}, f\right\rangle \\
& =\sum_{\ell=1}^{2 N-1} \sum_{j \in \mathbb{Z}} \sum_{\lambda \in \Lambda}\left\langle f, \tilde{\psi}_{\ell, j, \lambda}\right\rangle\left\langle\psi_{\ell, j, \lambda}, f\right\rangle
\end{aligned}
$$




$$
\begin{aligned}
& \leq\left(\sum_{\ell=1}^{2 N-1} \sum_{j \in \mathbb{Z}} \sum_{\lambda \in \Lambda}\left|\left\langle f, \tilde{\psi}_{\ell, j, \lambda}\right\rangle\right|^{2}\right)^{1 / 2}\left(\sum_{\ell=1}^{2 N-1} \sum_{j \in \mathbb{Z}} \sum_{\lambda \in \Lambda}\left|\left\langle f, \psi_{\ell, j, \lambda}\right\rangle\right|^{2}\right)^{1 / 2} \\
& \leq(\tilde{B})^{1 / 2}\|f\|_{2}\left(\sum_{\ell=1}^{2 N-1} \sum_{j \in \mathbb{Z}} \sum_{\lambda \in \Lambda}\left|\left\langle f, \psi_{\ell, j, \lambda}\right\rangle\right|^{2}\right)^{1 / 2} .
\end{aligned}
$$

Hence,

$$
\frac{1}{\tilde{B}}\|f\|_{2}^{2} \leq \sum_{\ell=1}^{2 N-1} \sum_{j \in \mathbb{Z}} \sum_{\lambda \in \Lambda}\left|\left\langle f, \psi_{\ell, j, \lambda}\right\rangle\right|^{2} .
$$

Same is the case for dual one. This completes the proof.

\section{Acknowledgements}

This Work is supported by the Start-Up Research Grant provided by UGC, Govt. of India.

\section{References}

[1] B. Behera and Q. Jahan, Biorthogonal wavelets on local fields of positive characteristic, Comm. Math. Anal., 15 (2013), 52-75.

[2] M. Bownik and G. Garrigos, Biorthogonal wavelets, MRA's and shift-invariant spaces, Studia Mathematica, 160 (2004), 231-248.

[3] A. Calogero and G. Garrigos, A characterization of wavelet families arising from biorthogonal MRA's of multiplicity d, J. Geom. Anal., 11 (2001), 187-217.

[4] A. Cohen and I. Daubechies, A stability criterion for biorthogonal wavelet bases and their related subband coding scheme, Duke Math. J., 68 (1992), 313-335.

[5] A. Cohen, I. Daubechies and J. C. Feauveau, Biorthogonal bases of compactly supported wavelets, Comm. Pure Appl. Math., 45 (1992), 485-560.

[6] L. Debnath and F. A. Shah, Wavelet Transforms and Their Applications, Birkhäuser, New York, 2015.

[7] I. Daubechies, Ten Lectures on Wavelets, SIAM: Philadelphia, 1992.

[8] M. Frazier, G. Garrigos, K. Wang and G. Weiss, A Characterization of functions that generate wavelet and related expansions, J. of Fourier Ana. and Appl., 3 (1997), 883-906.

[9] J. P. Gabardo and M. Z. Nashed, Nonuniform multiresolution analysis and spectral pairs, J. Funct. Anal., 158 (1998), 209-241.

[10] J. P. Gabardo and X. Yu, Wavelets associated with nonuniform multiresolution analysis and one-dimensional spectral pairs, J. Math. Anal. Appl., 323(2006), 798-817.

[11] E. Hernanez, X. Wang, G. Weiss, Smoothing minimally supportes frequency wavelets. I, J. Fourier Anal. Appl., 2 (1996), 329-340.

[12] E. Hernanez, X. Wang, G. Weiss, Smoothing minimally supportes frequency wavelets. II, J. Fourier Anal. Appl., 3 (1996), 23-41.

[13] H. O. Kim, R. Y. Kim and J. K. Lim, Characterizations of biorthogonal wavelets which are associated with biorthogonal multiresolution analyses, Appl. Comput. Harmon. Anal., 11 (2001), 263-272.

[14] R. Long and D. Chen, Biorthogonal wavelet bases on $\mathbb{R}^{d}$, Appl. Comput. Harmon. Anal., 2 (1995), $230-242$.

[15] F. A. Shah and Abdullah, Nonuniform multiresolution analysis on local fields of positive characteristic, Comp. Anal. Opert. Theory., 9(2015), 1589-1608.

[16] F. A. Shah and M. Y. Bhat, Vector-valued nonuniform multiresolution analysis on local fields, Int. J. Wavelets, Multiresolut. Inf. Process., 13(2015), No.4, Article Id: 1550029. 
[17] F. A. Shah and M. Y. Bhat, Nonuniform wavelet packets on local fields of positive characteristic, Filomat, 6 (2017), 1491-1505.

Department of Mathematics, Islamic University of Science and Technology, Awantipora, Pulwama-192122, Jammu and Kashmir, India.

E-mail: gyounusg@gmail.com 